\title{
Effect of age on contrast sensitivity function: uniocular and binocular findings
}

\author{
J E ROSS, ' D D CLARKE, ${ }^{2}$ AND A J BRON' \\ From the 'Nuffield Laboratory of Ophthalmology, University of Oxford, and the \\ ${ }^{2}$ Departmental of Experimental Psychology, University of Oxford, Oxford
}

SUMMARY Monocular and binocular contrast sensitivity function for a range of spatial frequencies was measured in two groups of subjects with normal vision. Statistically significant differences in performance between the younger group (age 20-30 years) and the older group (age 50-87 years) were found at all spatial frequencies sampled between 0.40 and $19.25 \mathrm{cpd}$. In the age range 50-87 years there was a linear decline in contrast sensitivity with age for medium and high spatial frequencies, but sensitivity for low spatial frequencies was independent of age.

In recent years a number of investigators have used contrast sensitivity function (CSF) as a supplementary measure of visual performance both in normal subjects' 2 and in patients..$^{3-5}$ Contrast thresholds are measured for a number of different spatial frequencies (the number of repetitions of a grating pattern, usually sinusoidal, within a unit of distance), and the results are represented graphically by plotting the reciprocal of the contrast threshold (contrast sensitivity) as a function of spatial frequency. In this way the performance of the eye can be specified over a wide working range and gives a broader description of visual function than the more conventional tests of visual acuity. Measurement of contrast sensitivity has revealed visual loss in patients with cerebral lesions, ${ }^{6}$ multiple sclerosis,${ }^{37}$ macular disease, ${ }^{8}$ glaucoma, ${ }^{+56}$ cataract," and amblyopia."

A number of the above disorders are prevalent among older patients in whom age related changes in the eye are likely to influence the results of visual function tests. It is thought that age related changes, such as those found with visual acuity measurement, are attributable not only to lenticular yellowing and senile miosis but also to neuronal and receptor loss in the visual pathway. ${ }^{12}$ The implication that the decline with age in human visual performance is the result of loss of neuronal quality and quantity receives support from other sources. Age related changes in the morphology of human cones ${ }^{13}$ and a decrease in the neuron population density of the macular projection

Correspondence to Dr J E Ross, Nufficld Laboratory of Ophthalmology, Walton Strect, Oxford OX2 6AW. areas in the visual cortex ${ }^{14}$ have been reported. In a tachistoscopic study Walsh ${ }^{15}$ has shown very clearly that there is a decrease in the efficiency of central processing of visual images with age.

Recently age dependent losses of contrast sensitivity have been reported by investigators who have used gratings displayed on an oscilloscope ${ }^{4}{ }^{16-210}$ and the Arden printed test. ${ }^{921}{ }^{22}$ Unfortunately the results of these studies do not all agree. Some investigators report a loss of contrast sensitivity for all spatial frequencies, ${ }^{9} 1921$ while others found losses for high and medium spatial frequencies only. ${ }^{16172022}$ One group found losses only at low and medium spatial frequencies. ${ }^{18}$ It is likely that this lack of agreement between studies is attributable to a number of procedural differences, to the type of equipment used, and to the small sample sizes in the majority or studies.

Four of the studies investigated visual performance in patients of more than 70 years. ${ }^{9}{ }^{18-20}$ Undoubtedly the study of older normal subjects poses a number of difficulties for the investigator and a definition of 'normal' becomes more difficult with increasing age. However, the incidence rates for disorders such as cataract, glaucoma, and macular disease in which abnormal CSF has been reported increase after the age of 65 years, ${ }^{2.3}$ so that this is an age group for which it is particularly important to obtain normative data in order to be able to evaluate visual dysfunction in relation to age. We have measured CSF in 70 subjects with normal eyes to obtain age norms for clinical use and to form a database for studies of various eye 
disorders. Fifty three of these subjects were aged 50 or more. The remaining 17 subjects were young adults who were included for comparison with the older group.

\section{Materials and methods}

Subjects. Seventy Caucasians aged between 20 and 87 years volunteered to take part in the study. None of the subjects had any previous experience in contrast sensitivity measurement techniques. The responses for two different age groups were examined. In the younger age group there were 17 subjects: 7 males, mean age 23 years (range $22-26$ years), and 10 females, mean age 25 years (range $20-30$ years). In the older group there were 53 subjects: 24 males, mean age 71 years (range 54-81 years), and 29 females, mean age 73 years (range $50-87$ years).

Subjects were included in the study if: (a) There was no current eye disorder on clinical examination. (b) There was no previous history of eye disorder that might affect visual function, for example, amblyopia. (c) There was no family history of glaucoma or diabetes. (d) There was visual acuity of $6 / 9$ (decimal $0 \cdot 66$ ) or better in each eye separately. (e) Optical correction was between -6 dioptres and +6 dioptres inclusive.

Cataract was excluded on the basis of the presence of a black lens opacity silhouetted against the red reflex on ophthalmoscopic examination. Any eye in which such an opacity was observed was excluded from the study. Severity of nuclear sclerosis was assessed in the slit beam on a scale of one to three. A score of one indicated the least sclerosis, and a score of three indicated the most sclerosis. Subjects with a score of two or less in each eye separately were included in the sample.

Glaucoma was excluded on the basis of a careful family history, examination of the optic disc, and applanation tonometry for those aged 70 or more. Subjects with intraocular pressure of less than 21 $\mathrm{mmHg}$ were considered to be non-glaucomatous. Senile macular degeneration was excluded on the basis of a morphological absence of macular degenerative change. All but seven of the subjects had visual acuity of $6 / 6(1.00)$ or better in each eye. These seven were subjects in the older age group: five had visual acuity of 6/9 (0.66) in one eye and 6/6 $(1.00)$ or better in the fellow eye, and two subjects aged 81 and 87 had visual acuity of 6/12 (0.50) in one and $6 / 9(0.66)$ in the other. The two eyes with visual acuity of $6 / 12(0.50)$ were excluded from the study. The apparent pupil size for all eyes was greater than $2.5 \mathrm{~mm}$.

Apparatus. Distance visual acuity was measured at $6 \mathrm{~m}$ by means of an internally illuminated Snellen chart that conformed to the British standard 4274. ${ }^{24}$ The luminance of the chart was $125 \mathrm{~cd} / \mathrm{m}^{2}$. A forced choice method was used to obtain visual acuity measurements.

Stationary vertical sine wave gratings of variable spatial frequency were generated on a display oscilloscope by a two channel computer addressed microprocessor waveform generator. ${ }^{25}$ The contrast of the grating pattern was adjusted by a computer linked attenuator. Contrast is defined as $\left(\left(\mathrm{L}_{\max }-\mathrm{L}_{\min }\right) /\left(\mathrm{L}_{\operatorname{mix}}\right.\right.$ $\left.+L_{\min }\right)$ ) where $L_{\max }$ and $L_{\min }$ are the maximum and minimum luminances respectively of the grating bars. A device wired in parallel with the microprocessor permitted the contrast of sinewave gratings to be faded on to the oscilloscope screen over a period of $0.5 \mathrm{~s}$ before the preprogrammed contrast level was achieved. This device overcomes the problem of stimulating transient mechanisms in the visual system at stimulus onset. ${ }^{26}$

The stimulus area on the oscilloscope screen was rectangular, $30 \mathrm{~cm} \times 20 \mathrm{~cm}$, with a matt grey surround extending out to form an area $35 \mathrm{~cm} \times 42 \mathrm{~cm}$. The mean screen luminance was $300 \mathrm{~cd} / \mathrm{m}^{2}$ and was independent of the contrast of the spatial frequency being displayed. The frame rate was $100 \mathrm{~Hz}$. Contrast values were linear for the six spatial frequencies which were used $(0 \cdot 4,0.95,2 \cdot 88,6 \cdot 73,12 \cdot 70,19.23$ cycles per degree of visual angle (cpd)). Changes in contrast of the sinewave gratings and the spatial frequency were either made interactively with the computer or preprogrammed. The subject viewed the screen from a distance of $280 \mathrm{~cm}$ and rested his chin on an adjustable rest. The test was described, and the subject was asked to respond to the presence of a vertical grating pattern, however faint, by pressing a hand held buzzer.

A preliminary routine was employed with the use of the interactive mode to establish the approximate range of threshold values for the six spatial frequencies. The gratings were presented on the screen in a pseudo random order at various contrast levels. This sequence provided an opportunity for the subject to become familiar with the grating patterns. An estimation of the contrast range in which the subject's threshold was likely to fall for each spatial frequency was obtained from these measurements and recorded. For each spatial frequency two levels of contrast were used to begin a double staircase sequence. One contrast value was above the predicted contrast threshold, and one contrast value was below.

After the preliminary trial had been completed two preprogrammed sequences were used: a double staircase technique, ${ }^{27}$ and the "up-down transformed response rule' (UDTR) technique. ${ }^{2 x}$ The contrast level at which each double staircase was begun was 


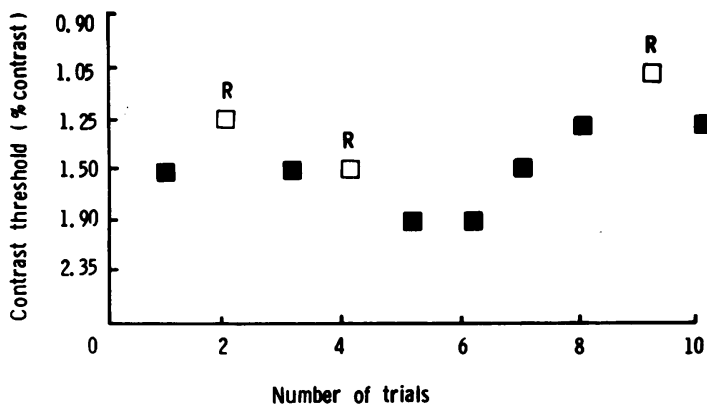

Fig. 1 A series of responses for the UDTR rule. The change in contrast level is represented on the vertical axis. The trial number is shown on the horizontal axis, and three reversals $\mathbf{R}$ are shown. The average of the values of the contrast at three reversals gives the value of contrast threshold. $\square=$ positive response. $\square=$ negative response.

determined by the response recorded in the preliminary trial. The choice of staircase represented on a given trial was made randomly by the computer. For the double staircase the contrast was increased after a negative response and decreased after a positive response. The contrast increment or decrement step was calculated for each presentation as a fraction $(0.079)$ of the previous contrast values.

When the crossover point on the double staircase had been reached a single sequence of stimuli for each spatial frequency value was then presented to the subject (the UDTR rule). The sequence began at the level of the last recorded values for each of the two staircases corresponding to a given spatial frequency. As with the double staircase the contrast was varied in steps of a predetermined size $(0.039)$, but no change in contrast level was made until one of the following patterns of results was obtained at any level:

$$
\begin{gathered}
\text { Decrease contrast after: } \\
\text { YYY or OYY } \\
\text { Y= positive response }
\end{gathered}
$$

$$
\begin{gathered}
\text { Increase contrast after: } \\
\text { YYO, OYO, OOO, or YOO } \\
\text { O=ncgative response }
\end{gathered}
$$

This set of responses converges on the stimulus level that gives approximately $70 \%$ of positive responses (Fig. 1). The threshold was computed from the mean of three reversals.

CSF measurements were made for the right, left, and both eyes of each subject. For the monocular viewing condition the non-viewing eye was covered with an eye patch. In order to assess the reliability of measurements 20 of the subjects $(10$ males and 10 females) were retested approximately 24 hours after the initial test session.

\section{Results}

Monocular and binocular CSF results for the two groups were characterised by a smooth curve which
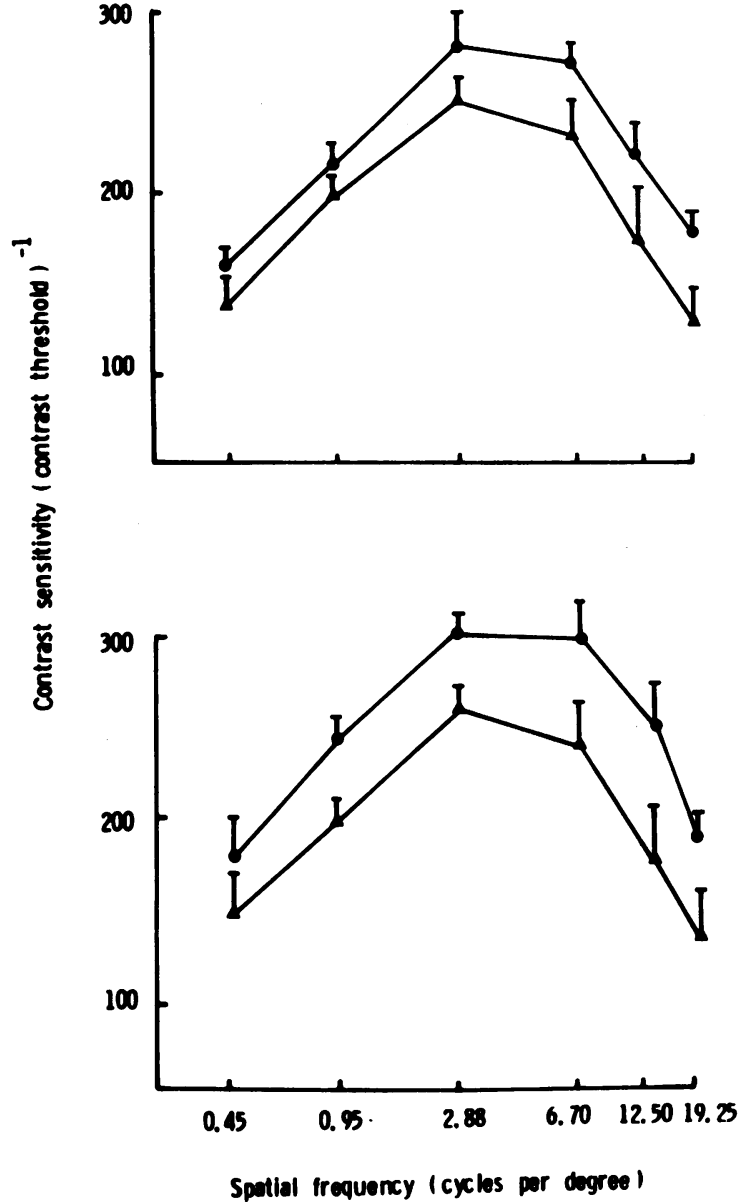

Fig. 2 Mean contrast sensitivity plotted as a function of spatial frequency for young $(\mathbf{O})$ and old $(\Delta)$ observers. The top graph shows values for monocular viewing, and the bottom graph for binocular viewing. The vertical bars represent \pm I $S D$.

peaked between 2 and $5 \mathrm{cpd}$ and was typically attenuated at low and high spatial frequencies (Fig. 2). Monocular sensitivity was consistently lower than binocular sensitivity for all spatial frequencies. No statistically significant sex differences were found, and so data for male and female subjects. were analysed together. In order to assess the reliability of responses the correlation coefficients derived from CSF values on a test-retest basis were examined. For each spatial frequency the correlation between the two test sessions exceeded $0.80(p<0 \cdot 001)$.

A one way analysis of variance was performed to determine whether the age related contrast sensitivity differences between the younger and older groups of observers were statistically significant (Fig. 2 ). For both viewing conditions there were significant 
Table 1 Contrast sensitivity function: one way analysis of variance between young and old observers for monocular and binocular viewing

\begin{tabular}{|c|c|c|c|c|c|c|c|c|}
\hline \multirow{2}{*}{$\begin{array}{l}\text { Spatial } \\
\text { frequency } \\
\text { (cpd) }\end{array}$} & \multicolumn{3}{|c|}{ Subjects (age 50-87) } & \multicolumn{3}{|c|}{ Subjects (age 20-30) } & \multirow[b]{2}{*}{ Fvalue } & \multirow[b]{2}{*}{ Tail p } \\
\hline & $\begin{array}{l}\text { Group } \\
\text { mean value }\end{array}$ & $S D$ & $\begin{array}{l}\text { Coefficient } \\
\text { of variation } \\
(S D / \text { mean } \times 100)\end{array}$ & $\begin{array}{l}\text { Group } \\
\text { mean value }\end{array}$ & $S D$ & $\begin{array}{l}\text { Coefficient } \\
\text { of variation } \\
(S D / \text { mean } \times 100)\end{array}$ & & \\
\hline \multicolumn{9}{|c|}{ Monocular vicwing } \\
\hline 0.40 & 1.435 & $0 \cdot 187$ & $13 \cdot 000$ & 1.648 & $0 \cdot 148$ & 8.980 & $29 \cdot 89$ & $<() \cdot(0) 1$ \\
\hline 0.95 & $2 \cdot 051$ & 0.173 & $8 \cdot 386$ & $2 \cdot 152$ & $0 \cdot 174$ & $8 \cdot 080$ & $6 \cdot 66$ & $0 \cdot(01$ \\
\hline $2 \cdot 88$ & $2 \cdot 542$ & 0.215 & 8.457 & $2 \cdot 811$ & $0 \cdot 204$ & $7 \cdot 250$ & $34 \cdot 31$ & $<() \cdot(\mathbf{0}) 1$ \\
\hline $6 \cdot 73$ & $2 \cdot 284$ & 0.312 & $13 \cdot 660$ & $2 \cdot 740$ & $0 \cdot 196$ & $7 \cdot 153$ & $54 \cdot 62$ & $<0 \cdot(001$ \\
\hline $12 \cdot 70$ & 1.736 & $0 \cdot 380$ & $21 \cdot 880$ & $2 \cdot 219$ & $0 \cdot 260$ & $11 \cdot 710$ & $39 \cdot 88$ & $<0 \cdot(0) 1$ \\
\hline $19 \cdot 25$ & $1 \cdot 252$ & $0 \cdot 284$ & $22 \cdot 680$ & $1 \cdot 784$ & $0 \cdot 135$ & $7 \cdot 560$ & $98 \cdot 21$ & $<0 \cdot(00)$ \\
\hline \multicolumn{9}{|c|}{ Binocular viewing } \\
\hline $0 \cdot 40$ & 1.494 & $0 \cdot 212$ & $14 \cdot 190$ & $1 \cdot 871$ & $0 \cdot 212$ & $11 \cdot 330$ & $61 \cdot 71$ & $<0 \cdot(0) 1$ \\
\hline 0.95 & $2 \cdot 098$ & $0 \cdot 171$ & $8 \cdot 150$ & $2 \cdot 376$ & $0 \cdot 142$ & 5.976 & $109 \cdot 43$ & $<() \cdot(0) 1$ \\
\hline $2 \cdot 88$ & $2 \cdot 630$ & $0 \cdot 222$ & $8 \cdot 440$ & $3 \cdot 139$ & $0 \cdot 230$ & $7 \cdot 327$ & $100 \cdot 84$ & $<0 \cdot(0) 1$ \\
\hline $6 \cdot 73$ & $2 \cdot 382$ & 0.291 & $12 \cdot 216$ & $3 \cdot 012$ & 0.235 & $7 \cdot 8(02$ & $106 \cdot 01$ & $<() \cdot(x) 1$ \\
\hline $12 \cdot 70$ & 1.836 & 0.344 & $18 \cdot 736$ & $2 \cdot 522$ & 0.244 & 9.674 & 96.65 & $<0 \cdot(001$ \\
\hline $19 \cdot 25$ & $1 \cdot 350$ & $0 \cdot 310$ & 22.960 & 1.934 & $0 \cdot 152$ & $7 \cdot 859$ & $97 \cdot 88$ & $<0 \cdot(0) 1$ \\
\hline
\end{tabular}

Table 2 Regression analysis of monocular contrast sensitivity on age

\begin{tabular}{|c|c|c|c|c|c|c|}
\hline CPD & $0 \cdot 40$ & 0.95 & $2 \cdot 88$ & $6 \cdot 73$ & $12 \cdot 70$ & $19 \cdot 25$ \\
\hline Slope & $-(0 \cdot() 0307$ & $-0 \cdot 00285$ & $-0 \cdot 01203$ & $-0 \cdot 01472$ & $-(0 \cdot(02017$ & $-(0 \cdot() 1578$ \\
\hline Intercept 50 year & $1 \cdot 50$ & $2 \cdot 10$ & $2 \cdot 80$ & $2 \cdot 66$ & $2 \cdot 20$ & 1.61 \\
\hline \multicolumn{7}{|c|}{ Pearson product corrclation } \\
\hline $\mathbf{r}$ & $-0 \cdot 137$ & $-(0 \cdot 139$ & -0.486 & $-0 \cdot 393$ & $-(0 \cdot 443$ & -0.464 \\
\hline $\mathrm{p}$ & $>0 \cdot(05$ & $>0 \cdot 05$ & $<0 \cdot 05$ & $<0 \cdot(05$ & $<(0 \cdot() 5$ & $<0 \cdot 05$ \\
\hline
\end{tabular}

differences among the mean values for the age groups for each spatial frequency (Table 1). Standard deviations for the younger group remained relatively constant over the range of spatial frequencies sampled and were smaller than the standard deviations for the older group. In the older group the standard deviations were greatest for the higher spatial frequencies. The coefficient of variation (the standard deviation of the distribution expressed as a percentage of the mean of the distribution for each spatial frequency) emphasised the greater variability in the frequency distribution in the older subjects.

The responses of the older age group were examined in more detail. A linear regression analysis of contrast sensitivity on age was made for all spatial frequencies. There was a significant decrease in contrast sensitivity with age for all but the two lowest spatial frequencies. The amount of scatter around the regression line was similar for monocular and binocular viewing, the least amount of scatter being found for a spatial frequency of $2 \cdot 88 \mathrm{cpd}$ in both cases (Table 2).

The older patients were divided into five age groups in order to examine the change with age in visual acuity measurement. However, entry to the study required visual acuity in each eye of $6 / 9$ or better, and so the range of acuities sampled was necessarily limited. The mean visual acuity values for each age group are given in Table 3. There was a statistically significant difference among the means of the five age groups. The main effect for age was significant $(F(4,99)=16 \cdot 40, p<0 \cdot 01)$. The relationship between a given level of visual acuity $(6 / 5,(1 \cdot 2)$, $6 / 6(1.00), 6 / 7.5(0 \cdot 80)$, or $6 / 9(0 \cdot 66))$ and contrast sensitivity function was examined. A one way analysis of variance was performed, and no significant difference was found among the mean CSF values for the four acuity levels. However, for the highest two spatial frequencies there was a rank order fall of contrast sensitivity with each level of visual acuity.

Table 3 Mean visual acuity values for older subjects

\begin{tabular}{lll}
\hline $\begin{array}{l}\text { Age group } \\
\text { (years) }\end{array}$ & $\begin{array}{l}\text { Group mean: log } \\
\text { minimum angle of } \\
\text { resolution }\end{array}$ & $\begin{array}{l}\text { Snellen } \\
\text { equivalent }\end{array}$ \\
\hline $48-60 n=20$ cycs & $1 \cdot 9$ & $6 / 5$ \\
$61-70 n=22$ eycs & $0 \cdot()$ & $6 / 6$ \\
$71-75 n=18$ cycs & $0 \cdot(0) 73$ & $6 / 6$ \\
$76-80 n=24$ cyes & $0 \cdot() 4$ & $>6 / 7 \cdot 5$ \\
81 and over $n=20$ cycs & $0 \cdot 10$ & $6 / 7 \cdot 5$ \\
\hline
\end{tabular}




\section{Discussion}

We measured contrast sensitivity function in two groups of normal subjects, first, to make a comparison of results between the older and the younger subjects, and second, to examine the pattern of results over a broad age range in 53 older subjects. The study was conducted to obtain age norms for clinical use and to form a database for studies of various eye disorders. In addition to monocular testing binocular assessments were performed in order to relate the results of visual function tests to visual performance as part of a wider study. ${ }^{29}$

The results showed that older observers had reduced contrast sensitivity for all spatial frequencies when compared with their younger counterparts. This was particularly marked for medium and high spatial frequencies. In the age range 50 to 87 years there was a linear decline in CSF with age for medium and high spatial frequencies. Within this age range the responses to low spatial frequencies appeared to be independent of age. These findings are in close agreement with many of the existing data obtained using oscilloscope generated gratings ${ }^{1617}$ and printed tests, ${ }^{19} 22$ but are opposed to those of Sekuler and Hutman, ${ }^{18}$ who found low but not high spatial frequency changes with increasing age.

Vaegan and Halliday ${ }^{9}$ consider that the most important reason for Sekuler and Hutman's ${ }^{1 \times}$ contrary findings is that their elderly sample was not representative, because 10 subjects had $6 / 6(1 \cdot 00)$ vision or better in the tested eye. They argue that the subjects came from a group with particularly high visual acuity for the age range who do not have the same visual performance for lower spatial frequencies. However, the older subjects used in the present study by reason of selection criteria suffer from the same bias, in that the observed Snellen visual acuities are better than one might expect in a representative sample of people in the older age range. Yet no age dependent changes for low spatial frequencies were observed. In a more recent study by the same group using a larger sample ${ }^{20}$ the authors found that sensitivity to medium and high spatial frequencies decreased with age after about 40 years. As the same method to determine contrast thresholds was used in both studies, it is possible that the age dependent low frequency attenuation of sensitivity in the first study ${ }^{18}$ is attributable to biases introduced by using a small population sample.

Thus there appears to be little evidence for preferential sensitivity losses at low spatial frequencies with aging. It is well known that degradation of any mechanical system, such as radios or hi-fi sets, results primarily in the loss of high frequency information. The visual system reacts in a similar manner to degraded images. ${ }^{30}$ It is the low spatial frequency information which conveys the major information about everyday objects as has been demonstrated in studies of face recognition ${ }^{30.31}$ and letter recognition..$^{32}$ So while it seems likely that aging changes at cellular level occur for neurones mediating both high and low spatial frequency information, these changes are less likely to show themselves in response to low frequency stimuli.

The question whether senile miosis contributes to the reduction of CSF with age has been raised by a number of investigators. ${ }^{17-19}$ Derefeldt, Lennerstrand, and Lundh ${ }^{17}$ do not give pupil sizes in their publication, but in the studies of Sekuler and Hutman ${ }^{18}$ and McGrath and Morrison ${ }^{19}$ the pupil sizes were greater than $2 \mathrm{~mm}$ as were those in the present study. Senile miosis may not affect contrast sensitivity as such. Other contributory factors may be involved. However, Woodhouse ${ }^{33}$ has shown that in the presence of clear optical media, and with screen luminance values above $34 \mathrm{~cd} / \mathrm{m}^{2}$, the difference between spatial frequencies resolved at pupil diameters between 2 and $4 \mathrm{~mm}$ is minimal at both high $(0.97)$ and low $(0 \cdot 11)$ contrasts. In the present study the subjects had clear media, the screen luminance was well above 34 $\mathrm{cd} / \mathrm{m}^{2}$, and the minimum pupil size was greater than $2.5 \mathrm{~mm}$. The limiting effects of diffraction can be dismissed at this pupil size, which suggests that in this study senile miosis is unlikely to be a contributory factor in the reduction of contrast sensitivity with age.

It appears from the present data that, in common with a number of other visual functions, CSF declines with age and in particular at the medium and high spatial frequency end of the curve. The existing data are commensurate with this finding, though lack of standardisation of stimuli and psychophysical procedures from laboratory to laboratory has resulted in variations as to the magnitude and exact location of the sensitivity loss on the contrast sensitivity curve. Responses to stimuli at the low spatial frequency end of the spectrum were independent of age in the subjects over 50 years old in the present study and in the majority of other studies. When pathological status is assessed there are obvious advantages for the use of stimuli which are relatively immune to aging effects in the visual system. An added advantage of the use of low spatial frequencies is that the effects of optical aberration are minimal at this end of the CSF curve. ${ }^{1}$

There is great potential for the application of CSF testing in a number of visual disorders. In the present study a series of age norms is presented for six spatial frequencies with the use of a rigorous testing scheme. Though it is clear that different investigators will present findings based on the particular arrange- 
ments available to them, there is a need to standardise approaches in order to make CSF testing more generally useful and available.

This research was supported by grant No $81 / 2$ from Oxford Regional Health Authority.

\section{References}

1 Campbell FW, Green DG. Optical and retinal factors affecting visual resolution. J Physiol (Lond) 1965; 181: 576-93.

2 Banks MS, Salapatek P. Contrast sensitivity function of the infant visual system. Vision Res 1976; 16: 867-9.

3 Regan D, Silver R, Murray R. Visual acuity and contrast sensitivity in multiple sclerosis-hidden visual loss. Brain 1977; 100: 563-79.

4 Arden GB, Jacobson JJ. A simple grating test for contrast sensitivity: preliminary results indicate value in screening for glaucoma. Invest Ophthalmol Visual Sci 1978; 17: 23-32.

5 Atkin A, Bodis-Wollner I, Wolkstein M, Moss A, Podos S. Abnormalities of central contrast sensitivity in glaucoma $\mathrm{Am} \mathrm{J}$ Ophthalmol 1979; 88: 205-11.

6 Bodis-Wollner I, Diamond SP. The measurement of spatial contrast sensitivity in cases of blurred vision associated with cerebral lesions. Brain 1976; 99: 695-710.

7 Bodis Wollner I, Hendley CD, Mylin LH. Thornton J. Visual evoked potentials and the visuogram in multiple sclerosis. Ann Neurol 1979; 5: 40-7.

8 Sjostrand J, Frisen L. Contrast sensitivity in macular disease. Arch Ophthalmol 1977; 55: 507-14.

9 Vacgan, Halliday BL. A forced-choice test improves clinical contrast sensitivity testing. BrJ Ophthalmol 1982; 66: 477-91.

10 Hess RF, Woo G. Vision through cataracts. Invest Ophthalmol Visual Sci 1978; 17: 428-35.

11 Hess RF. Contrast sensitivity assessment of functional amblyopia in humans. Trans Ophthalmol Soc UK 1979; 99: 391-97.

12 Weale RA. Senile changes in visual acuity. Trans Ophthalmol Soc UK 1975; 95 : 36-8.

13 Marshall J. Ageing changes in human cones. Proceedings of the 23rd International Congress of Ophthalmology, Kyoto, May 1978. Amsterdam: Excerpta Medica, 1978: 1: 375-8.

14 Devaney KO, Johnson HA. Neuron loss in the aging visua cortex of man. J Gerontol 1980; 35: 836-41.

15 Walsh DA. Age differences in central perceptual processes. A dichoptic backward masking investigation. J Gerontol 1976; 31: 178-85.
16 Arundale $K$. An investigation into the variation of human contrast sensitivity with age and ocular pathology. BrJ Ophthalmol 1978; 62: 213-5.

17 Derefeldt G, Lennerstrand G, Lundh B. Age variations in normal human contrast sensitivity. Acta Ophthalmol (Kbh) 1979; 57: 679-90.

18 Sckuler R. Hutman LP. Spatial vision and aging 1. Contrast sensitivity J Gerontol 1980; 35: 692-9.

19 McGrath C, Morrison JD. The effects of age on spatial frequency perception in human subjects $Q J$ Exp Physiol 1981; 66: 253-61.

20 Owsley C, Sekuler R, Siemsen D. Contrast sensitivity throughout adulthood Vision Res 1983; 23: 689-99.

21 Skalka HW. Effect of age on Arden grating acuity $\mathrm{BrJ}$ Ophthalmol 1980; 64: 21-3.

22 Sokol S, Domar A, Moskowitz A. Utility of the Arden grating test in glaucoma screening: high false positive rate in normals over 50 years of age. Invest Ophthalmol Visual Sci 1980; 19: 1529-33.

23 Department of Health and Social Security. Blindness and partial sight in England 1969-1976. London: HMSO, 1979. Reports on public health and medical subjects No 129.

24 British Standards Institution. Specifications for test charts for determining distance visual acuity. British Standard Specification 4274,1968 .

25 Taylor P. A microprocessor controlled gratings generator. J Physiol (Lond) 1978; 284: 20P-1P.

26 Kulikowski JJ, Tolhurst DJ. Psychophysical evidence for sustained and transient detectors in human vision. J Physiol (Lond) 1973; 232: 149-62.

27 Cornsweet T. The staircase method in psychophysics $\mathrm{Am} \mathrm{J}$ Psychol 1962; 75: 485-91.

28 Wetherill GB. Sequential estimation of quantal response curves. $J$ R Statist Soc Series B 1963; 25: 1-48.

29 Ross JE. The functional effects of visual disorder. DPhil thesis, Oxford University 1983; (Unpublished).

30) Ginsburg AP. Specifying relevant spatial information for image evaluation and display design. An explanation of how we sec certain objects. Proc Soc Information Display 1980: 21: 219-27.

31 Owskley C, Sckuler R, Boldt C. Aging and low-contrast vision: face perception. Invest Ophthalmol Visual Sci 1981; 21:362-5.

32 Ginsburg AP. Relationship between the detection and recognition of Snellen letters and contrast sensitivity for normal and abnormal visual systems. J Opt Soc Am 1978; 68: 1455.

33 Woodhouse JM. The effect of pupil size on grating detection at various contrast levels. Vision Res 1975; 15: 645-8.

34 Green DG, Campbell FW. Effect of focus on the visual response to a sinusoidally modulated spatial stimulus. J Opt Soc Am 1965; 55: $1154-7$. 